Revista Eureka sobre Enseñanza y Divulgación de las Ciencias

ISSN: 1697-011X

revista.eureka@uca.es

Universidad de Cádiz

España

\title{
El rol docente y la indagación científica: análisis de una experiencia sobre plagas en una escuela vulnerable de Chile
}

Morales, Marlene; Acosta, Katherine; Rodríguez, Carlos

El rol docente y la indagación científica: análisis de una experiencia sobre plagas en una escuela vulnerable de Chile

Revista Eureka sobre Enseñanza y Divulgación de las Ciencias, vol. 19, núm. 2, 2022

Universidad de Cádiz, España

Disponible en: https://www.redalyc.org/articulo.oa?id=92069718002

DOI: https://doi.org/10.25267/Rev_Eureka_ensen_divulg_cienc.2022.v19.i2.2201 


\section{El rol docente y la indagación científica: análisis de una experiencia sobre plagas en una escuela vulnerable de Chile}

Teacher's role and scientific inquiry: Analysis of an experience about pests in a Chilean rural school

Marlene Morales

Departamento de Quimica. Universidad de Tarapacá.

Arica. Chile, Chile

mmorales@uta.cl

iD https://orcid.org/0000-0002-0915-8547

\section{Katherine Acosta}

Departamento de Educación. Universidad de Tarapacá.

Arica. Chile, Chile

kacosta@academicos.uta.cl

iD https://orcid.org/0000-0001-5744-6618

Carlos Rodríguez

Escuela Dario Salas Diaz. Arica. Chile, Chile

carlosrodriguezcisterna@gmail.com

iD https://orcid.org/0000-0002-1962-7335
DOI: https://doi.org/10.25267/

Rev_Eureka_ensen_divulg_cienc.2022.v19.i2.2201

Redalyc: https://www.redalyc.org/articulo.oa? $\mathrm{id}=92069718002$

Recepción: 13 Mayo 2021

Revisado: 03 Agosto 2021

Aprobación: 17 Enero 2022

\section{ReSUMEN:}

La incorporación de la indagación científica en las aulas rurales plantea importantes desafíos para los docentes que la orientan. En el presente artículo se analiza la importancia del rol del docente en una actividad de indagación científica. Para ello, se realizó el análisis del caso de la Mitigación de la mosquita blanca en árboles de ficus, una experiencia indagatoria llevada a cabo con 22 estudiantes de sexto a octavo año de Educación General Básica de una escuela rural vulnerable en Chile. Los resultados respaldan que los aprendizajes de los estudiantes a través de la indagación demandan una orientación y apoyo adecuados por parte del docente. Asimismo, se revela que la investigación científica escolar puede emerger en contextos rurales poco favorecidos a través de la indagación.

Palabras Clave: Rol Docente, Indagación Científica, Escuela Rural, Educación en Ciencias, Educación Básica.

\section{Aвstract:}

The incorporation of the scientific inquiry in rural science classrooms brings up important challenges for the teachers leading them. This current paper analyzes the importance of the teacher's role in a scientific inquiry activity. Therefore, an analysis was carried out in the case of the Mitigation of the whitefly in the ficus tree. This inquiring experience was applied with 22 sixth to eighth grade elementary students from a vulnerable rural school in Chile. The main results support that the students' learning through inquiry demands a guidance and an adequate support offered by the teacher. Besides, the case study shows how the inquiry-based science education can emerge from deprived rural contexts through inquiry.

KEYWORDS: Teaching Role, Scientific Inquiry, Rural School, Science Education, Elementary School.

\section{INTRODUCCIÓN}

A nivel internacional las grandes metas de la educación plantean cuestiones importantes acerca de qué tipo de enseñanza de las ciencias mejora la calidad de los aprendizajes, facilita el desarrollo de competencias 
científicas y habilita la formación de ciudadanos alfabetizados científicamente (Roberts 2007). Por ello, cada vez son más las investigaciones enfocadas en describir el impacto de la indagación científica (IC) en la educación en ciencias de los estudiantes alrededor del mundo (Ariza 2017; Bevins y Price 2016; Lederman $e t$ al.2013). En tal sentido, el nuevo marco curricular en Estados Unidos enfatiza claramente que los estudiantes experimenten mediante el diseño y la realización de actividades indagatorias con el fin de aprender sobre la ciencia y cómo esta se desarrolla (NGSS Lead States 2013), así como también sobre su epistemología (NRC 2012).

Si pensamos en la formación de estudiantes en la sociedad del conocimiento y en la cantidad de información que hoy debemos procesar, lo más sugerente es enseñar la indagación como medio para aprender ciencia, para que estos se apropien del lenguaje científico a través de la experimentación (Lederman et al. 2013). Llevar a cabo una IC es posible en un aula escolar, aunque con algunas salvedades (Bybee 2006; Crawford 2014). Por ejemplo, la ciencia escolar no suele contar con un alto grado de sofisticación, lo que afecta al uso de equipos tecnológicos o al alcance y duración de sus investigaciones. Sin embargo, sus semejanzas metodológicas posibilitan que los estudiantes reflexionen de manera similar a los científicos, se involucren en experiencias indagatorias y desarrollen conocimientos sobre los fundamentos de la ciencia (NGSS Lead States 2013). Durante el aprendizaje por indagación, el estudiante adquiere un conocimiento profundo sobre los tópicos tratados, aunque no necesariamente un dominio amplio de todos los contenidos evaluables (Harlen 2010; McConney et al. 2014). Esto último sugiere que es en la profundidad de la comprensión y calidad del aprendizaje del estudiante sobre un determinado tema, y en las habilidades científicas desarrolladas, en donde la indagación cobra gran relevancia.

Bevins y Price (2016) señalan que la efectividad de la IC en la enseñanza de las ciencias se consolida a través de un amplio repertorio de trabajos empíricos e investigaciones que reportan resultados de aprendizaje positivos para los estudiantes en aspectos como rendimiento, motivación y desarrollo de habilidades científicas. En ese sentido, Putambekar y Kolodner (2005) han señalado que las actividades de indagación implican que el profesorado desarrolle diversas estrategias para ser un guía adecuado de los estudiantes. Una de estas estrategias corresponde al andamiaje, entendido como una variedad de estrategias de enseñanza utilizadas por el profesor que busca en los estudiantes mayor compresión, profundidad e independencia en el proceso de aprendizaje (Parra et al. 2018), y en donde el apoyo sobre cómo realizar la tarea y la reflexión sobre por qué la tarea debe resolverse de una forma determinada es parte clave del proceso (Hmelo-Silver et al. 2007).

A partir de lo anterior, es válido suponer que la orientación de aprender indagando supone grandes desafíos tanto para profesores como para estudiantes (Krajcik et al. 2001). Por lo cual, no es factible describir cómo se producen los aprendizajes de los estudiantes a través de la IC sin entender el rol del docente en dicho proceso. Sin embargo, ¿cuál es el rol del docente en la IC? Estudios como los de Holbrook y Kolodner (2000) proponen que se deben ejecutar cuatro acciones para construir un proceso de andamiaje en la IC: a) Problematizar la tarea mediante la formulación de preguntas relevantes y la proposición de soluciones iniciales; b) Registrar los temas a investigar con el fin de realizar procesos de retroalimentación colectivos; c) Proponer la construcción de diseños de investigación, y d) Socializar los diseños del alumnado a sus pares. No obstante, son escasas las experiencias que permiten evidenciar con ejemplos concretos dicha intervención docente. En consecuencia, este artículo presenta una experiencia de indagación donde un profesor y sus estudiantes realizan una actividad de IC en el marco de la educación científica a nivel básico y rural. En los apartados se relatan los momentos pedagógicos relevantes de la experiencia indagatoria que describen las acciones adoptadas por el docente durante el proceso y las consecuentes acciones realizadas por los estudiantes. 


\section{Fundamento Teórico}

Ariza et al. (2016) señalan que la NRC define la IC como:

«Una actividad polifacética que incluye la observación, la formulación de preguntas, la búsqueda de información en libros y otras fuentes para conocer lo que ya se sabe sobre un tema, el diseño y planificación de investigaciones, la revisión de ideas atendiendo a la evidencia experimental disponible, el manejo de herramientas asociadas a la adquisición, análisis e interpretación de datos, la formulación de respuestas, explicaciones, predicciones y la comunicación de resultados». (p. 300)

Asimismo, la IC es un proceso en el cual «se plantean preguntas acerca del mundo natural, se generan hipótesis, se diseña una investigación, se colectan y analizan datos con el fin de encontrar una solución al problema» (Windschitl 2003, p. 113). La comunidad científica emplea estos procesos en la construcción de conocimiento científico, de modo que se asume que esta forma de trabajo también debería tener un protagonismo importante en el aula en la generación de conocimiento científico escolar, por lo que, desde una perspectiva sociocultural, la indagación es entendida como un enfoque pedagógico, lo cual significa que también es un proceso de orientación hacia la reflexión en el proceso de enseñanza de las ciencias (Abell et al. 2006).

Desarrollar un proceso de IC requiere promover el desarrollo de habilidades en los estudiantes. Así, algunas de las destrezas mencionadas por Bybee (2006) son: reconocer qué preguntas pueden ser respondidas al realizar una investigación científica; esquematizar y guiar una investigación científica; utilizar instrumentos y procedimientos apropiados para reunir, examinar y deducir datos; pensar razonable y objetivamente para relacionar las explicaciones con los resultados; extender reseñas, aclaraciones, pronósticos y modelos coherentes con las pruebas obtenidas. Desde el modelo propuesto por Harlen (2015) se entiende que la IC invita al desarrollo progresivo de diferentes ideas científicas a partir de la investigación escolar realizada, lo cual favorece la construcción del conocimiento y la comprensión de los fenómenos naturales. En ese sentido, el trabajo del profesor consiste en apoyar a los estudiantes a desarrollar las capacidades necesarias para hacer una investigación científica, es decir, fortalecer las habilidades de indagación. Para el caso de la indagación guiada el profesor plantea los cuestionamientos que serán la guía para la investigación, contrario a lo que se plantea en la indagación abierta donde es el estudiante quien diseña todo el protocolo de investigación, lo cual incluye la pregunta, procedimiento y conclusiones (Martin-Hansen 2002). En ambos casos, la guía docente resulta fundamental, el andamiaje que el profesorado planifique para promover los aprendizajes, independientemente de quién elabore las pautas para desarrollar la investigación. Por otro lado, es importante mencionar la relevancia que tiene la utilización del contexto cercano para la enseñanza de las ciencias, lo que permite fomentar actitudes positivas hacia las ciencias (Bennett $e t$ al. 2007).

\section{Metodología}

Con el fin de afrontar el objetivo del trabajo, se presenta un estudio de caso de una experiencia indagatoria liderada por un profesor de Ciencias y realizada por 22 estudiantes (10 niños y 12 niñas) de sexto a octavo año ( $\bar{x}=12$ años) de Educación General Básica de una escuela rural vulnerable ubicada al norte de Chile. Cabe mencionar que el término vulnerable hace referencia al indicador utilizado por el Programa de Alimentación Escolar perteneciente a la Junta Nacional de Auxilio Escolar y Becas (JUNAEB s.f.), el cual busca medir el riesgo de deserción escolar de un establecimiento utilizando como criterio para su evaluación el factor socioeconómico de sus estudiantes. La muestra de estudiantes participó de un taller extracurricular de su escuela llamado Academia de Ciencias, dirigido por el mismo docente. El caso o la experiencia indagatoria titulada Mitigación de la mosquita blanca en árboles de ficus fue registrada mediante la observación activa empleando un diario de campo y recopilando los trabajos y actividades del alumnado. Esta se resume a continuación, para posteriormente y con más detalle, mediante la presentación de nueve 
momentos pedagógicos (ver figura 1), describir cómo cada acción del estudiante demanda acciones específicas por parte del docente. En paralelo, también se mencionan los procedimientos y acciones realizadas por el profesor para conducir las preguntas derivadas de la curiosidad, el entorno inmediato y las necesidades de los estudiantes.

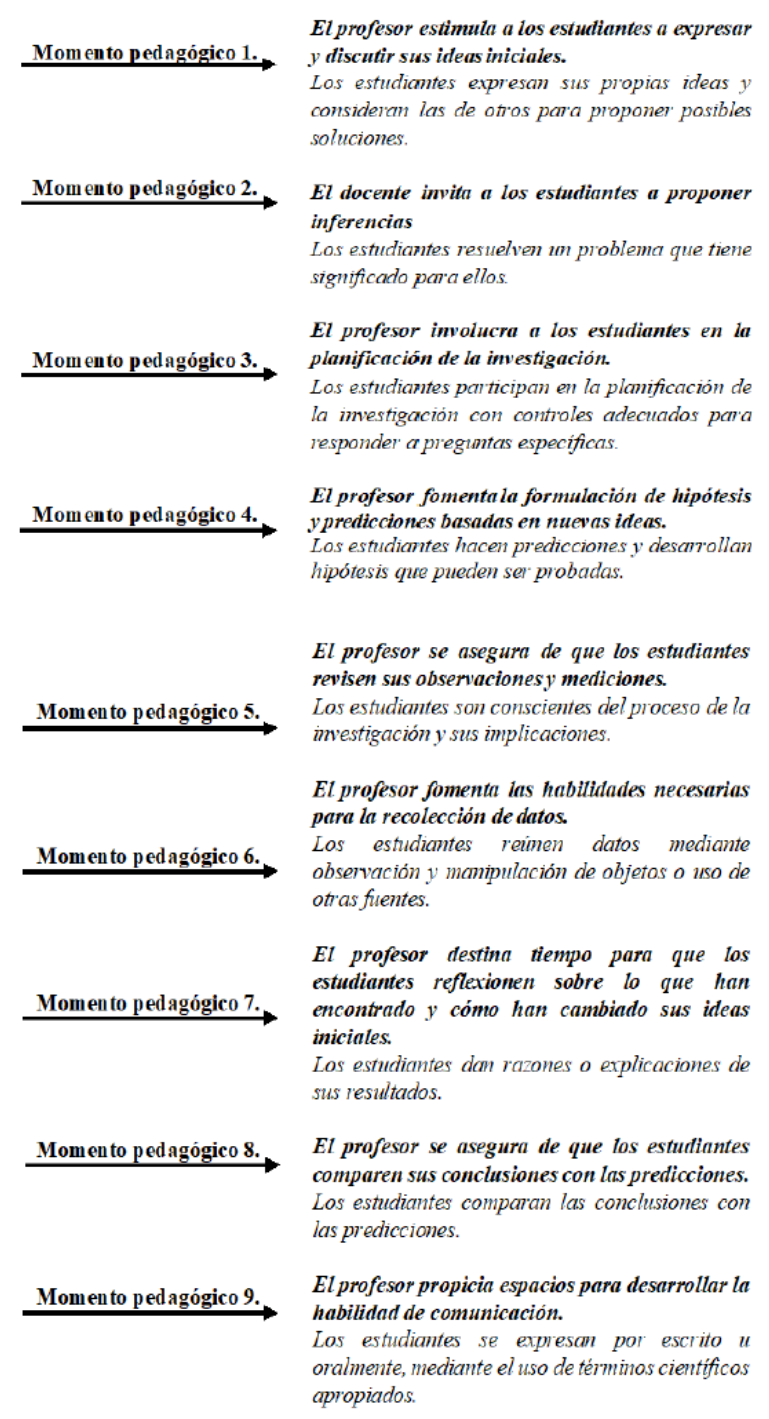

FIGURA 1

Momentos pedagógicos y acciones docente-estudiante que emergen durante el proceso educativo.

\section{Contexto de la experiencia}

Los participantes de esta experiencia son parte de una institución de tipo rural que ha incorporado dentro de su proyecto educativo la IC como enfoque pedagógico y, en paralelo, el desarrollo de habilidades científicas como uno de sus sellos institucionales. La escuela se ubica en una zona rural agrícola en las afueras de la ciudad de Arica, Chile. La institución desarrolla el Proyecto de Educación Intercultural Bilingüe que responde a un contexto donde el $93,5 \%$ de los estudiantes declara pertenecer a un pueblo originario, mayoritariamente aymara. Por su parte, un tercio del total de la matrícula corresponde a estudiantes extranjeros provenientes de Perú y Bolivia. En paralelo, en los últimos 10 años su índice de vulnerabilidad ha tenido un promedio del $91,64 \%$, lo cual la categoriza como una escuela con alto índice de vulnerabilidad. 
A pesar de este dato, la escuela se ha posicionado como una de las mejores de la región. En especial, la práctica en ciencias ha sido destacada como una de las 15 mejores por la Agencia de Calidad de la Educación en Chile (Agencia de Calidad de la Educación 2019).

En el valle de Azapa (Arica) se explota ampliamente la agricultura, siendo esta la principal fuente laboral de los padres de los estudiantes que componen la escuela. El control de plagas en esta región se hace principalmente a base de insecticidas químicos que a largo plazo generan resistencias en los insectos o plagas y, a su vez, se convierten en contaminantes del agua y algunos tipos de flora (FAO 2013). Dentro de las plagas con mayor importancia, tanto en el área agrícola como ornamental, se encuentra la mosquita blanca del cocotero Aleurodicus sp. (Hemíptera: Aleyrodidae) que afecta a un amplio espectro de especies.

\section{DESARROLLO DE LA EXPERIENCIA INDAGATORIA}

\section{Momento pedagógico 1. A partir de un problema, el profesor estimula a los estudiantes a plantearse preguntas e identificar aquellas que pueden investigarse}

Desde hace algún tiempo que la comunidad educativa manifestaba alergias a un polvillo blanco que transportaba el viento. Asimismo, los estudiantes se percataban de que algunas plantas de su escuela tenían problemas en las hojas. Ellos, al ser hijos de padres agricultores, conocían las características de las plantas cuando estas presentaban signos de enfermedad, por lo que, durante una clase de la Academia de Ciencias, mientras observaban estomas, le preguntaron al profesor: «¿Qué es el polvillo blanco presente en las plantas de la escuela?». En ese momento, el docente identificó la pregunta de sus estudiantes como una oportunidad para desarrollar una actividad indagatoria. En consecuencia, contestó al interrogante con otras preguntas: «¿Será este el causante de las alergias en las personas? ¿Será esto lo que afecta las plantas de la escuela? ¿Cómo se puede ayudar a remediarlo?». El profesor animó a los estudiantes a dar respuesta a estas preguntas y convirtió este estudio en el tema central de la academia. La decisión se basó en que la actividad indagatoria promueve en los estudiantes el desarrollo de procedimientos, actitudes y contenidos que son la base del currículum nacional de Ciencias, además de ser una buena oportunidad para intentar dar solución a un problema propio del contexto local por medio de una actividad de investigación.

\section{Momento pedagógico 2. El profesor invita a los estudiantes a proponer explicaciones preliminares}

En una primera instancia el docente aprovechó la oportunidad de aprendizaje que generaban las preguntas de sus estudiantes e invitó a la formulación de inferencias sobre lo que posiblemente les podría estar ocurriendo a las plantas enfermas. En ese sentido, los estudiantes infirieron que esto ocurría por exceso o falta de riego, deficiencia de nutrientes, problemas con el $\mathrm{pH}$ del suelo y/o del agua o por la influencia de virus, hongos o insectos. Las explicaciones preliminares que el profesor extrajo de los estudiantes le fueron muy útiles para conocer sus ideas previas, aspecto fundamental que le permitió evaluar el progreso de las explicaciones de los estudiantes y, posteriormente, del aprendizaje derivado del proceso de investigación.

\section{Momento pedagógico 3. El profesor involucra a los estudiantes en la planificación de la investigación}

Cuando los estudiantes se enfrentan a una situación de investigación tienen que tomar decisiones para precisar los problemas; esto involucra definirlos, identificarlos y entenderlos. El rol del profesor es 
fundamental para el logro de este objetivo, sugiriendo como primera medida la fundamentación teórica. Durante esta etapa los estudiantes leyeron e identificaron mediante información botánica las características morfológicas que presentan las plantas cuando se ven afectadas por deshidratación, alteraciones del $\mathrm{pH}$, carencia de nutrientes o presencia de plagas. Posterior a la documentación, el profesor invitó a los estudiantes a realizar un inventario de todas las plantas presentes en la escuela, determinando taxonómicamente cada una de ellas (ver tabla 1). Con el fin de desarrollar habilidades de observación y recolección de datos, además de poner a disposición instrumentos apropiados para obtener información sobre el tipo de vegetación afectada y las características que presenta dicha condición, se realizó un perfil de vegetación de las plantas presentes en toda la escuela. Para ello, los estudiantes trabajaron en cuatro grupos por perfil de vegetación. La figura 2 muestra los perfiles de vegetación que se dibujaron, caracterizaron y describieron de cada planta presente por sector con el registro de su altura y sus características foliares. También, muestra la enumeración de las plantas que presentan algún signo de daño y sus posibles causas según fuentes bibliográficas.

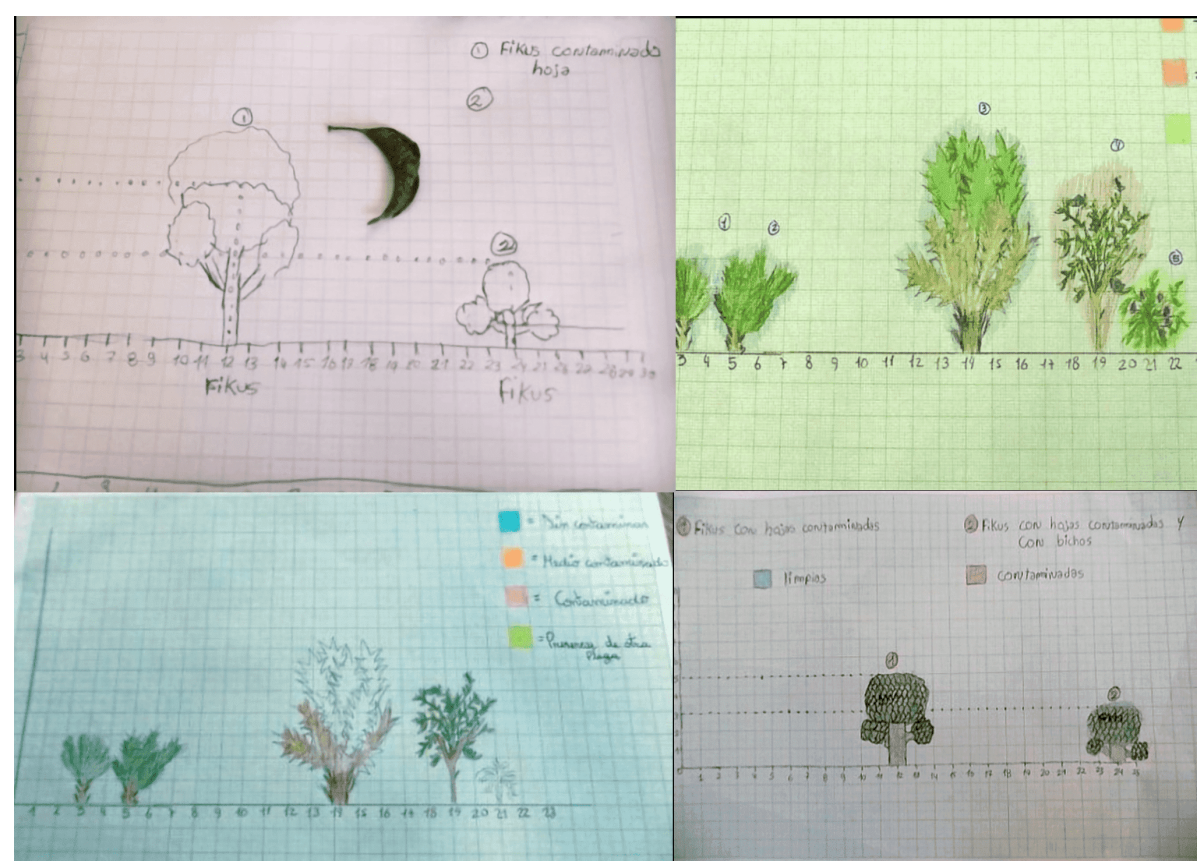

FIGURA 2

Perfiles de vegetación por sectores horizontales representados por dibujos hechos por el alumnado. 
TABLA 1

Inventario de las plantas infectadas en la escuela.

\begin{tabular}{|l|c|c|l|}
\hline \multicolumn{1}{|c|}{ Planta } & N. ${ }^{\circ}$ de individuos & $\begin{array}{c}\text { Tipo de plaga } \\
\text { Mosquita blanca } \\
2=\text { Piojillo del ficus }\end{array}$ & Parte afectada de la planta \\
\hline Palmeras. Phoenix dactyliferaalta & 4 & 1 & Envés de las hojas \\
\hline Ficus. Ficus benjamina & 4 & 1 y & Envés de las hojas \\
\hline Aloe Vera. Aloe barbadensis & 12 & Ninguna & Ninguna \\
\hline Guanábano. Annona muricata & 1 & 1 & Envés de las hojas \\
\hline Mango. Mangifera indica & 2 & 1 & Haz de las hojas \\
\hline Pino. Pinus nigra & 1 & Ninguna & Ninguna \\
\hline Rosal. Rosa sp. & 5 & 1 & Haz y envés de las hojas \\
\hline Chañar. Geoffroea decorticans & 4 & Haz de las hojas \\
\hline Laurel. Laurus nobilis & 1 & Envés de las hojas \\
\hline
\end{tabular}

Después de crear el perfil de vegetación de la escuela, los estudiantes mantuvieron sus inferencias iniciales sobre la posibilidad de alteraciones en el $\mathrm{pH}$ del suelo dado que el agua de riego proviene de napas subterráneas, de modo que se plantea la necesidad de determinar el $\mathrm{pH}$ tanto del agua como del suelo de todos los sectores donde se ubican las plantas. Para realizar esta acción uno de los apoderados que trabaja en agricultura les enseñó a medir el $\mathrm{pH}$ del agua mediante el uso de instrumentos digitales (pH-metro portátil). Esta práctica fue realizada en la escuela por los estudiantes bajo la supervisión del apoderado y del profesor. Para la determinación del $\mathrm{pH}$ en suelo, el profesor optó por recurrir a las redes de apoyo con que cuenta la institución, en este caso, una universidad local.

Producto de la actividad experimental, el agua de riego presentó un $\mathrm{pH}$ de 6,8 y el suelo un rango de $\mathrm{pH}$ entre 6 y 6,5. Estos valores según la literatura son aptos para el desarrollo de las plantas (Ibarra et al. 2009), por lo que se descarta que la causa de la afectación de las plantas sea por problemas del pH del suelo o del agua de riego. Por otra parte, producto del perfil de vegetación y del análisis detallado de las plantas se logró identificar la presencia de un tipo de plaga que afecta a las plantas de la escuela, especialmente a los árboles de ficus: la mosca blanca del cocotero (ver figura 3). 


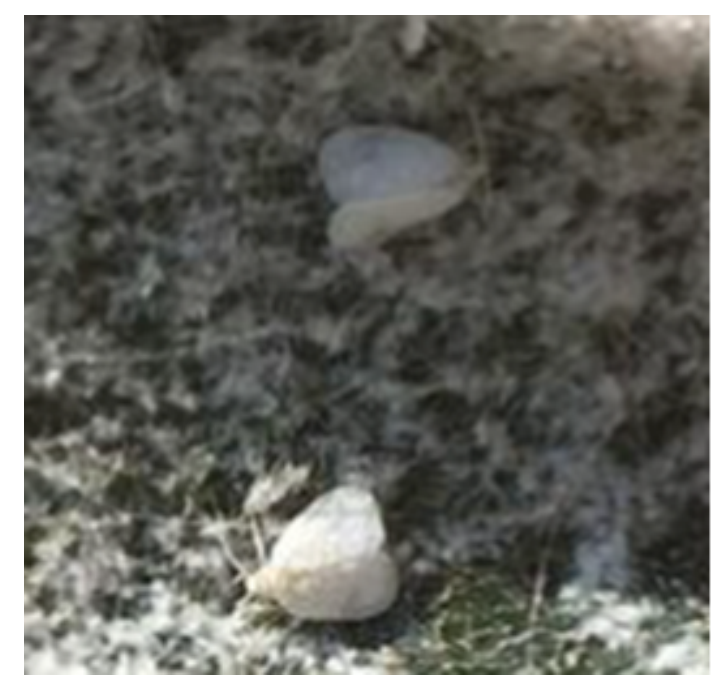

FIGURA 3

Mosca blanca del cocotero (Aleurodicus sp.).

De este análisis también se obtuvo que la melaza, la cual se desprende de las ninfas de la mosca blanca (figura 4), es lo que otorga el color blanco característico que presenta el envés de las hojas (INIA 2018).

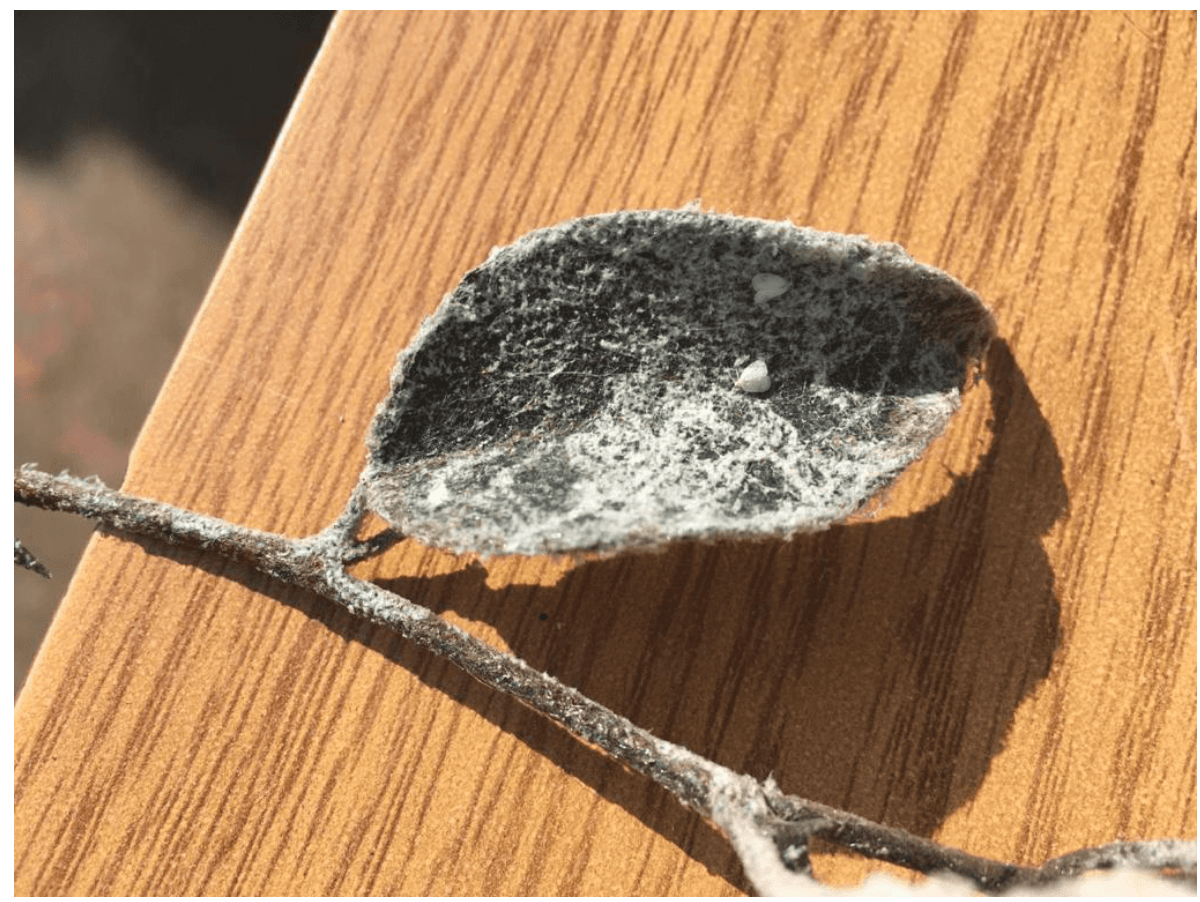

FIGURA 4

Melaza en hojas de ficus producto de las ninfas de la mosquita blanca.

Momento pedagógico 4. El profesor fomenta la identificación de las variables, la formulación de predicciones e hipótesis y la propuesta de un diseño experimental

Con los resultados obtenidos del inventario de las plantas y mediciones del $\mathrm{pH}$, el profesor induce a un nuevo momento de aprendizaje, invitando a los estudiantes a formular una conclusión a priori. Es así como el alumnado concluye que la mosquita blanca era la causa de afectación de las plantas. Asimismo, surgió la 
hipótesis de que la melaza producida por las ninfas se transporta por el viento, entra por los orificios olfativos y causa alergias en ciertas personas de la comunidad educativa.

Posteriormente, el profesor guía el análisis del perfil de vegetación hacia las plantas que no presentaron signos de infección. A raíz de ello, los estudiantes observaron que el aloe vera (Aloe barbarensis) y el pino (Pinus devoniana) fueron las únicas plantas dentro de la escuela que no presentaron contaminación por mosquita blanca (ver tabla 1). Por consiguiente, predijeron que un preparado de estas podría eventualmente eliminar la plaga al ser aplicado en otras plantas. Frente a este acontecimiento el profesor decidió guiar a sus estudiantes en la elaboración de macerados a base de dichas plantas y la realización de ensayos experimentales que permitieran comprobar, en condiciones in vitro, la eficacia y efectividad de estos preparados. Para poder comparar, el profesor sugirió a los estudiantes la utilización de algún otro preparado natural a base de plantas que anteriormente hubiera sido empleado en procesos de mitigación de la mosquita blanca y que posibilitara la comparación con los que elaborarían, en términos de eficacia en el control de plagas.

Tras completar la búsqueda decidieron emplear Chrysopogon zizanioides (pasto vetiver). Esta especie vegetal es un pasto de origen africano utilizado como fitorremediador de aguas y suelos que ha demostrado tener propiedades de repelencia por alcance en los cultivos. Esta información fue utilizada por los estudiantes para fines comparativos con los otros dos extractos. La fase experimental se desarrolló en condiciones in vitro, utilizando plantas de ficus (Ficus benjamina). La elección de la planta a tratar se debió principalmente a que esta presentaba el mayor porcentaje de contaminación de individuos por la plaga.

Otro momento importante en este proceso se asocia con el establecer relaciones entre las variables que habían sido previamente formuladas. Estas relaciones entre variables coinciden una vez más con los modelos explicativos del fenómeno en estudio y es un momento propicio para que los estudiantes den paso a su creatividad. El profesor encausó este momento hacia la construcción de hipótesis a partir de las concepciones que los y las estudiantes plantearon a través de observaciones, inferencias y predicciones, por ende, los invitó a poner de manifiesto de forma explícita la relación entre las variables que dieron cuerpo a la hipótesis de la investigación. Después de generar los espacios de discusión e intercambio de ideas, el docente guió a los estudiantes a tomar decisiones que permitieran dar paso a la siguiente etapa y a concretar la hipótesis final.

$\mathrm{H}$ : La densidad poblacional de ninfas de mosquita blanca disminuye cuando las plantas son tratadas con preparados a base de aloe o pino.

Una vez que los y las estudiantes lograron establecer las variables y su relación a través de la construcción de la hipótesis, el profesor estableció el diálogo que ayudó a determinar las condiciones y requerimientos apropiados para la comprobación de esta. Al igual que en el proceso anterior, la institución se vio obligada a acudir a sus redes por no contar con un laboratorio de ciencias que generara las condiciones apropiadas para la realización de los ensayos. Durante esta actividad, el grupo de estudiantes recibió orientación por parte del cuerpo académico de una universidad local, a quienes les plantearon su investigación científica y diseño experimental. La actividad experimental contó con un periodo de evaluación de los extractos de siete días. Los procedimientos empleados durante la fase experimental fueron los siguientes:

- Obtención de ninfas de Aleurodicus sp.: La recolección de las muestras biológicas se transformó en otra oportunidad de aprendizaje, la cual fue aprovechada por el docente para reforzar técnicas de extracción y normas de autocuidado. Estas muestras se obtuvieron de hojas de ficus (Ficus benjamina) presentes en la escuela.

- Elaboración de los extractos naturales: Una de las etapas de la metodología fue la preparación de los extractos, tarea realizada por el alumnado en condiciones de laboratorio bajo la supervisión de su profesor (ver figura 5). Dicha etapa fue planificada de esta manera con el fin de observar sobre el terreno la forma en la que trabajan los científicos. 


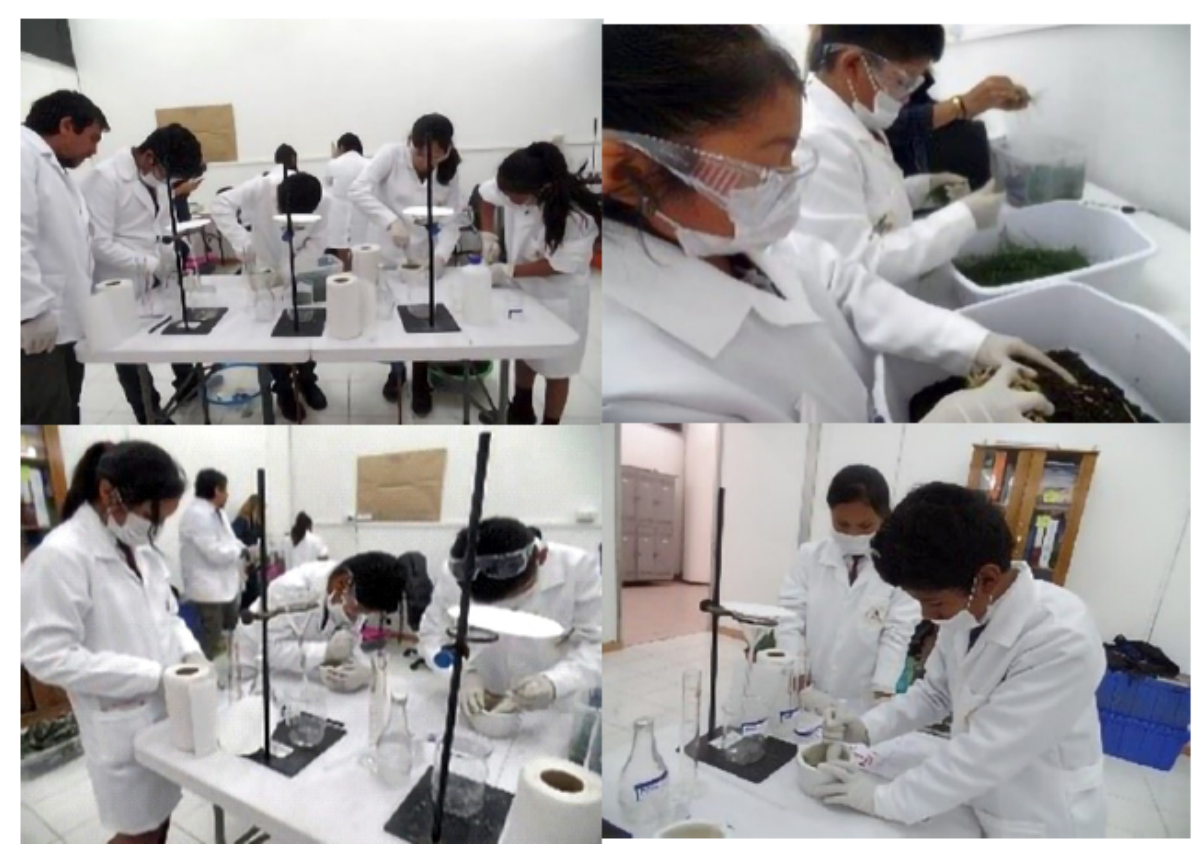

FIGURA 5

Estudiantes durante el proceso de elaboración de los extractos vegetales.

Una vez recolectadas las hojas infectadas, estas fueron analizadas en el laboratorio. La siguiente etapa consistió en definir el diseño experimental que se seguiría para la recolección de datos. Para esto el equipo conformado por el profesor e investigadores de la universidad guiaron al conjunto de estudiantes a definir una estrategia apropiada. Para cada una de las elaboraciones de los extractos los estudiantes maceraron 350 g de raíz de vetiver, aloe vera y pino con $450 \mathrm{~mL}$ de agua destilada, $250 \mathrm{~mL}$ de etanol al $70 \%$ y 2 g de jabón en barra para ropa, agitando hasta su completa disolución. Una vez filtrado, el extracto se trasvasijó a un recipiente oscuro almacenado en un refrigerador a una temperatura de $3{ }^{\circ} \mathrm{C}$ para retardar su degradación.

\section{Momento pedagógico 5. El profesor se asegura de que los estudiantes revisen sus observaciones y mediciones}

Los procesos de investigación escolar están cargados de momentos para el aprendizaje, desde lo más mínimo se puede construir una gran oportunidad de reflexión para el alumnado. Es así que el profesor, junto al equipo de investigadores, invitó a los y las estudiantes a realizar la última revisión de la metodología propuesta para la experimentación. Estos últimos, confiando en su diseño, no percibieron el error que el docente buscaba que identificaran. Es por esto que el profesor instruyó a sus estudiantes la importancia de evaluar la mortalidad natural que, en un ensayo, implica disponer de testigos no sometidos al tratamiento. El grupo de estudiantes llegó al siguiente diseño experimental al definir la realización de cuatro montajes experimentales: T1: Tratamiento con extracto de vetiver, T2: Tratamiento con extracto de pino, T3: Tratamiento con extracto de aloe y T4: control o testigo, sin tratamiento alguno. Sin embargo, no fue hasta la fase experimental cuando el profesor pudo constatar que sus estudiantes entendieron el significado de esta variable. Así el alumnado debió repetir la fase experimental, es decir, volver a hacer el conteo de ninfas, pero ahora considerando un grupo testigo como fuente de comparación. Con respecto al conteo de las ninfas vivas en el bioensayo (ver figura 6) se obtuvieron los siguientes datos en términos de días por cada tratamiento. 


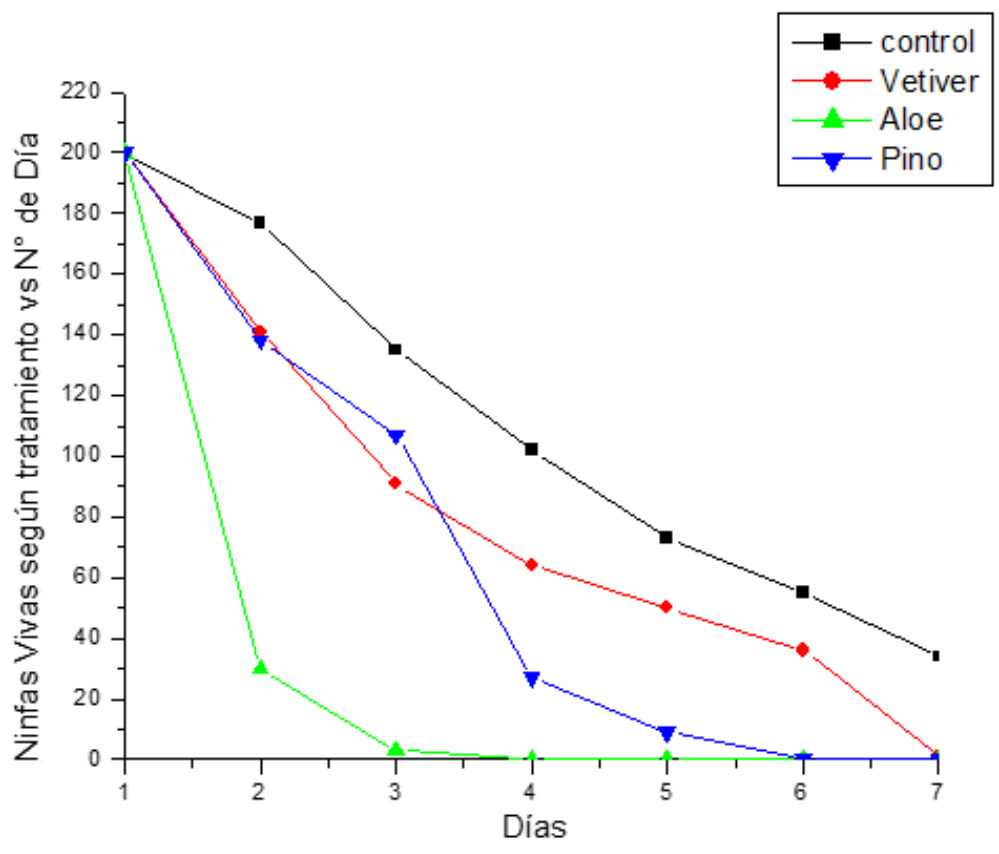

FIGURA 6

Ninfas vivas según tratamiento.

\section{Momento pedagógico 6. El profesor fomenta las habilidades necesarias para la recopilación de datos}

La observación es una de las etapas que produce gran asombro en los estudiantes y, si a estas observaciones les agregamos los registros que se pueden apreciar mediante la experimentación, se transforman en procesos de aprendizajes únicos para el alumnado. Los ensayos realizados por los y las estudiantes generaron una gran oportunidad para desarrollar habilidades de observación y recolección de datos. Respecto al conteo de ninfas en el bioensayo, se emplearon 200 ninfas por cada montaje, teniendo consigo un total de 800 individuos para el estudio (ver tabla 2). El conteo de la población de ninfas presentes en este bioensayo se realizó durante siete días consecutivos. Las hojas que contenían las ninfas fueron sumergidas durante 10 segundos en los extractos contenidos en vasos de precipitado correspondientes, sujetándolas con pinzas entomológicas. La mortalidad de las ninfas se determinó en cada día de tratamiento, observando directamente al estereoscopio. 
TABLA 2

Comparación de la eficacia en valor porcentual de cada tratamiento en función de los días de aplicación.

\begin{tabular}{|l|l|l|l|l|}
\hline Días & Control & Vetiver $\%$ Eficacia & Aloe $\%$ Eficacia & Pino $\%$ Eficacia \\
\hline 1 & 200 & $200 /(0,0)$ & $200 /(0,0)$ & $200 /(0,0)$ \\
\hline 2 & 177 & $141 /(20,4)$ & $30 /(83,1)$ & $138 /(22,1)$ \\
\hline 3 & 135 & $91 /(32,0)$ & $3 /(97,8)$ & $107 /(22,1)$ \\
\hline 4 & 102 & $64 /(37,3)$ & $0 /(100)$ & $27 /(73,6)$ \\
\hline 5 & 73 & $50 /(36,6)$ & $0 /(100)$ & $9 /(87,7)$ \\
\hline 6 & 55 & $36 /(34,6)$ & $0 /(100)$ & $0 /(100)$ \\
\hline 7 & 34 & $1 /(97,1)$ & $0 /(100)$ & $0 /(100)$ \\
\hline
\end{tabular}

Para el cálculo del porcentaje de mortalidad finita (variación del número de individuos, en ausencia de nacimientos, emigración o inmigración, en un periodo de tiempo) se empleó la siguiente fórmula:

$$
\text { Mortalidad finita } \% M=\left(\frac{N o-N t}{N o}\right) * 100
$$

[Ecuación 1]

Donde: $\mathrm{No}=$ Población inicial $\mathrm{Nt}=$ Población final \%M= Porcentaje de mortalidad

$$
\text { Eficacia }=\left[1-\left(\frac{N t}{N^{\prime} t}\right)\right] * 100
$$

[Ecuación 2]

Donde: $\mathrm{Nt}=$ Número de individuos en el lote tratado, al cabo de $\mathrm{t}$ días

$N^{\prime} t=$ Número de individuos en el lote testigo, al cabo de $t$ días

Una vez que los estudiantes lograron sistematizar los resultados de sus ensayos, los organizaron en tablas y gráficos y utilizaron ecuaciones recopiladas de las fuentes. Es necesario que ellas y ellos puedan racionalizar la información recolectada, de manera que sean capaces de construir el conocimiento científico al igual que lo hacen los científicos. Mejorar las destrezas cognitivas y del pensamiento del alumnado es una de las tareas fundamentales del profesorado de Ciencias. Poner énfasis en su razonamiento deductivo de los y las estudiantes utilizando las pruebas recabadas mediante los ensayos y la sistematización de los resultados resulta ser una gran oportunidad para iniciar el proceso de cierre del aprendizaje, y la argumentación en ciencias es un momento pedagógico oportuno para este proceso. 


\section{Momento pedagógico 7. El profesor destina tiempo para que los estudiantes reflexionen críticamente sobre lo que han encontrado y cómo han cambiado sus ideas iniciales}

El hablar de relaciones entre las hipótesis y los experimentos planteados resulta una gran estrategia para el logro de las destrezas de comunicación en ciencias. Todo lo anterior respalda la necesidad docente de establecer un momento que permita a sus estudiantes el análisis, la interpretación y argumentación de los resultados obtenidos. En la presente experiencia, la guía del profesor propició que sus estudiantes observaran los resultados organizados en tablas y gráficos para luego obtener las siguientes reflexiones: existen dos tratamientos que lograron disminuir significativamente la población de ninfas, el macerado a base de aloe y el macerado a base de pino. En la calidad de las hojas expuestas a este último se pudo evidenciar un notable desgaste en comparación con los otros tratamientos realizados. En consecuencia, los estudiantes infirieron que el extracto de pino ejerció un efecto negativo en la turgencia de las células de las hojas de ficus, ya que estas presentaban signos de deshidratación, donde además se pudo observar un color cobrizo en sus bordes, al contrario de lo que ocurrió con el macerado a base de aloe. Por ello, los estudiantes concluyeron que el extracto de aloe vera, por su eficacia y efectividad en este diseño experimental, es el mejor agente natural para el control de ninfas de Aleurodicus sp. presentes en hojas de ficus en condiciones de laboratorio. Con esta información se puede considerar la presencia de algún compuesto químico natural en las plantas de aloe vera con propiedades biocidas ante este tipo de plagas.

\section{Momento pedagógico 8. El profesor se asegura de que los estudiantes comparen sus conclusiones con las predicciones}

Con el fin de que sus estudiantes pudieran realizar el proceso de metacognición sobre lo planteado al inicio y el camino recorrido para el logro de los aprendizajes producto de la investigación, el profesor estableció como momento pedagógico el contraste entre las ideas iniciales y las finales, después de obtener las conclusiones al término de los ensayos y análisis de los resultados. Dicha etapa permite el desequilibrio cognitivo fundamental para cambiar las concepciones previas de los estudiantes, llevándolos a la construcción mental de una nueva idea y nuevos aprendizajes. Mediante este trabajo, los estudiantes junto a su profesor pudieron evidenciar la existencia de dos parásitos que afectan principalmente a las plantas que se encuentran en la escuela: los Piojitos del Ficus benjamina.Gynaikothrips Uzeli) y la mosquita blanca del cocotero (Aleurodicus sp). Asimismo, evidenciaron que este último era responsable de las alergias manifestadas por algunos integrantes de la comunidad educativa, producto de la melaza producida por algunos estadios de los insectos. Por último, llegaron al resultado de que el aloe vera presentó un mejor desempeño en la mitigación de la población de ninfas de mosquita blanca, Aleurodicus sp. (Hemíptera: Aleyrodidae) in vitro, encontradas en plantas de ficus. Este extracto demostró una altísima efectividad desde el cuarto día de aplicación, sobre el $25 \%$ con respecto al pino y vetiver. A su vez, el extracto de aloe vera no produjo la deshidratación de las hojas, como sí lo hizo el extracto de pino.

\section{Momento pedagógico 9. El profesor propicia espacios para desarrollar la habilidad de comunicación de los resultados}

Las experiencias de IC están encaminadas a que el alumnado tenga la oportunidad de elaborar informes, compartir resultados y aplicar situaciones nuevas a los conocimientos construidos durante el proceso de investigación. Con el objetivo de comunicar sus resultados, el docente motiva a sus estudiantes a participar en el evento que se realiza anualmente en la región, el Congreso Regional Escolar de la Ciencia y la Tecnología 
(CRECYT), en donde presentaron su investigación en la XII versión de este evento en la categoría Ciencias Naturales en Educación Básica, efectuada en el año 2017. La investigación fue seleccionada como la mejor en aquella categoría, ganando el primer premio. Debido a esto, los y las estudiantes tuvieron la oportunidad de divulgar sus hallazgos en otras instituciones educativas y contactar a un periódico local para la divulgación de los resultados principales.

\section{CONSIDERACIONES FINALES}

Aprender ciencias a través de la indagación puede ser un proceso mucho más significativo cuando se ejecuta en una comunidad de aprendices que desarrollan el conocimiento científico en el lugar donde este es aplicado, por ende, es importante mencionar la relevancia que tiene la utilización del contexto para el aprendizaje de las ciencias, para desarrollar una comprensión científica (Bennett et al. 2007). En este caso, las plantas presentes en la escuela y sus plagas fueron el escenario oportuno para el aprendizaje. La motivación por desarrollar la experiencia podría vincularse a la contextualización dada a la misma, ya que esta es el reflejo de las vivencias diarias de los estudiantes en casa con sus padres agricultores. La actividad, sin duda, permitió la experimentación suficiente para que los y las estudiantes vivieran de cerca la experiencia y así lograran aprendizajes significativos (Hagenauer et al. 2015). No obstante, se entiende que no se debe confundir estar involucrado y activo de manera física con estar motivado y activo de manera intelectual.

La experiencia se centra en promover un ambiente de aprendizaje colaborativo, donde el estudiante es inmerso en una cultura científica al involucrase en la tarea misma de la indagación. Como elemento relevante, es posible aseverar que el proceso desarrollado es un apoyo al aprendizaje colaborativo, donde el alumnado entiende con esta manera de trabajar que la ciencia es una construcción colectiva y que las investigaciones científicas son producto de la colaboración entre investigadores, de compartir un objetivo en común basado en la adquisición de roles y normas. Es así como al involucrarse o desarrollar su propia investigación científica bajo un enfoque indagatorio se promueve el trabajo autónomo y colaborativo entre estudiantes (Crawford 2014).

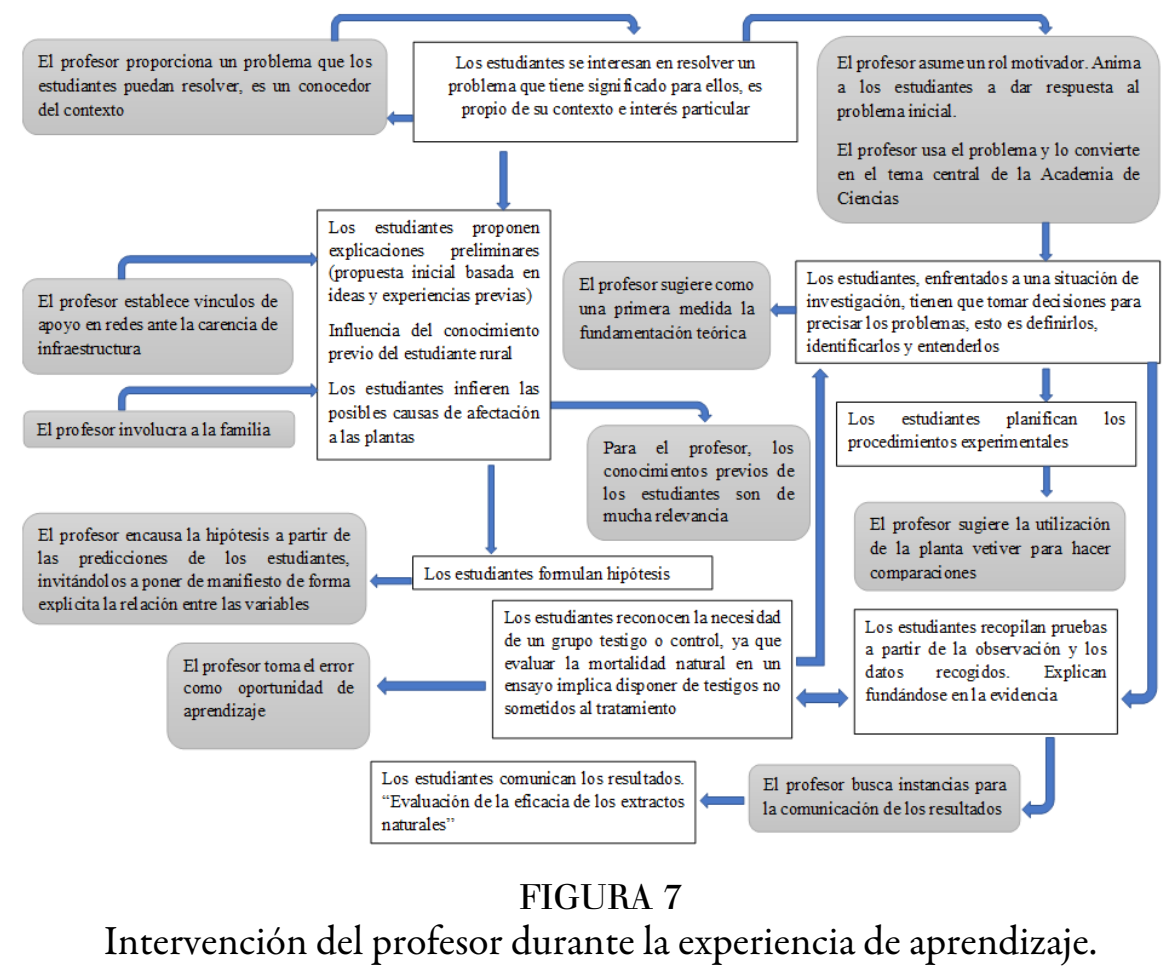


Como se puede evidenciar en la figura 7, el monitoreo del profesor es constante durante todo el proceso. Asimismo, es posible aseverar que la intervención del docente tiene un fin común: mantener la presencia del fenómeno (ver figura 8), en específico, limitar la complejidad del proceso incitando a sus estudiantes a formular preguntas durante toda la experiencia, las que además son diversas, tanto en el nivel cognitivo que conllevan (recordar, comprender, aplicar y evaluar) como en el tipo de conocimiento que solicitan (factual, conceptual, procedimental). A su vez, el docente interviene para generar un ambiente de diálogo, análisis y reflexión permanente, lo que favorece la construcción de competencias. De esta forma, entrega pistas de información de manera gradual, sintetiza hallazgos y ofrece una visión global del estado de la cuestión, elabora a partir de las ideas de los estudiantes, estableciendo relaciones con la vida cotidiana previas a la instrucción, identifica qué saben y cómo piensan los estudiantes, sugiere determinadas acciones y ofrece orientaciones sobre cómo realizar una determinada acción (González-Weil et al. 2012).
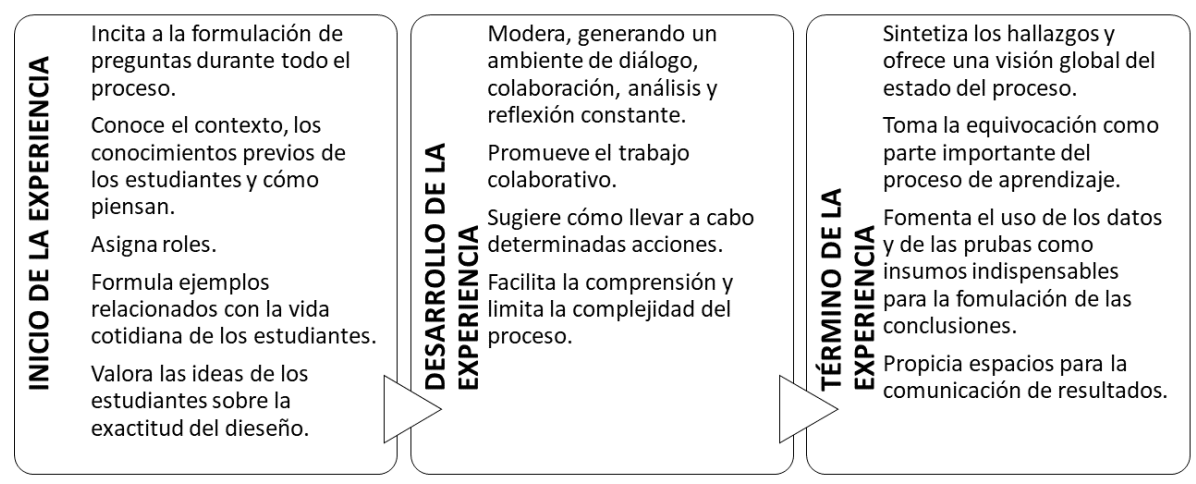

\section{FIGURA 8}

Rol del profesor durante la experiencia indagatoria.

Paralelamente a ello, el docente valora la opinión del alumnado sobre la exactitud del diseño experimental, algo que abre la posibilidad de equivocarse como parte sustancial del proceso de aprendizaje. En este caso en particular, cuando los y las estudiantes no toman en cuenta la necesidad de un grupo control. El docente dirige esta situación no mediante la penalización del error, sino que por el contrario hace que esto se convierta en una oportunidad para el aprendizaje.

Respecto a la intervención del profesor, es posible evidenciar a través de la experiencia las bondades de la indagación guiada en el logro de los aprendizajes. De acuerdo con las investigaciones consultadas, cuando el proceso está debidamente asistido por el profesor se obtienen beneficios importantes en materia de aprendizajes (Furtak et al. 2012; Lazonder y Harmsen 2016; Minner et al. 2010). Esto contrasta con la situación del alumnado que aprende a partir de demostraciones de laboratorio, donde todo se plantea a priori, o el aprendizaje a través de la indagación abierta, que es complejo por la necesidad de fijar la mirada en fenómenos de interés. De este modo, la indagación de tipo guiada permite al alumnado tener más oportunidades para familiarizarse con el objeto de su indagación y esta diferencia la enmarca el ambiente de la indagación, en este caso particular un ambiente propio del contexto del estudiante.

Siguiendo con este punto, indudablemente parte del resultado de esta experiencia se le atribuye al docente. Así es como los mejores resultados de enseñanza se asocian al profesor que considera las opiniones de sus estudiantes y se asegura de que participen activamente en el seguimiento de su propio aprendizaje y el desarrollo de sus propias habilidades cognitivas (Hattie 2009). En esa dirección, «los beneficios de la indagación sobre el aprendizaje del alumnado se ven fuertemente afectados por la forma en la que el docente planifica, articula y orienta el proceso» (Ariza 2017, p. 295). Como se aprecia en la figura 7, el rol del docente consistió en orientar a sus estudiantes, motivándolos a pensar y reflexionar durante todo el proceso, algo contrario a ciertas visiones críticas que surgen en torno a la IC (Kirschner et al. 2006; Mayer 2004). Sin 
embargo, en numerosas escuelas, aún en los casos en los que se ofrecen materiales y desarrollo profesional especializado, el profesorado encuentra dificultades importantes para la implementación de una indagación de calidad en el aula. Por consiguiente, es muy certero aseverar también que en gran medida el resultado de la experiencia lo aporta la cultura que se ha establecido en la escuela rural mencionada en el presente trabajo, ya que esta ha favorecido que tanto profesores como estudiantes adquieran una cultura indagatoria (Agencia de Calidad de la Educación 2019).

En esta institución las tareas de indagación están centradas en el interés del alumnado, quienes a menudo plantean la pregunta que guía su investigación, diseñan los procedimientos a seguir para contestar a su pregunta y sacan conclusiones derivadas de los resultados y las pruebas para, finalmente, comunicar los resultados. Esta es la aproximación más cercana a la experiencia del trabajo científico (Martin-Hansen 2002). Desde esta perspectiva, cobra mucha relevancia propiciar instancias para que los estudiantes se sumerjan en una cultura científica que les permita aprender mucho más (Busquets et al. 2016). Esto último, se puede alcanzar mediante las acciones deliberadas de las escuelas con el fin de crear oportunidades sistemáticas que permitan realizar investigaciones científicas (Treviño et al. 2009).

Desde el prisma de vulnerabilidad del contexto, podemos concluir que este enfoque, conjugado con un rol adecuado del docente, aporta a los estudiantes insumos que les permiten confiar en sí mismos, sentirse capaces de resolver problemas de su entorno y tener una opinión basada en pruebas ante las situaciones del contexto. La experiencia revela que la indagación puede emerger desde contextos rurales y poco favorecidos, lo que hace que sea indispensable conocer las características de las escuelas que podrían ayudar a mitigar el efecto de las desigualdades sociales sobre el logro académico de los estudiantes, y de los factores escolares que podrían mejorar este aspecto, aun en situaciones de desmedro socioeconómico. Desde esta perspectiva, las ciencias pueden ser una importante vía para acortar las brechas culturales entre estudiantes de familias más pobres y sus escuelas, mejorando las oportunidades de aprendizaje y movilidad social, lo cual solo es posible si el profesor juega un rol gravitante en la estructuración de las oportunidades de aprendizaje de la educación científica. Es así como desde la perspectiva de la investigación educativa resulta claro que el desarrollo de competencias científicas entre los estudiantes es indispensable para mejorar las oportunidades de aprendizaje. Por lo tanto, los componentes clave para mitigar el efecto que producen las desigualdades sociales sobre el logro académico son los buenos docentes y las escuelas que cumplen con sus responsabilidades en torno al aprendizaje de sus alumnos. Por consiguiente, si estos elementos claves estuviesen acompañados de políticas sociales y educativas que tengan como objetivo acortar las brechas que existen por la condición social y la segregación escolar en el aprendizaje, se podría ver engrandecido el potencial transformador de la educación (Treviño et al. 2009).

Si se pretende que la indagación en el aula repercuta en una mejora en la enseñanza de las ciencias, el o la docente debe diseñarla y articularla teniendo en mente los resultados de aprendizaje que se desean alcanzar, por ende, es importante plantear tareas de indagación con claros objetivos de aprendizaje. En concreto, se deben especificar los conceptos, habilidades y desempeños observables finales deseados para el alumnado, tarea que puede ser facilitada al tener claro cada propósito de la intervención docente (Chernicoff y Echeverría 2012).

Los resultados de aprendizaje del alumnado derivados de la IC dependen de la clase de actividades que se lleven a cabo en el aula y del nivel de guía o apoyo ofrecido por el profesorado. En ese sentido, el rol del docente en la IC se enfoca en regular y estructurar el fenómeno o proceso indagatorio, es decir, debe propiciar que el proceso indagatorio se lleve a cabo hasta el final. El profesor es quien rescata los intereses del alumnado, pero a su vez los guía, logrando transformar estas inquietudes en momentos de aprendizaje. Esto requiere que el docente sepa aprovechar el contexto para propiciar una enseñanza de las ciencias contextualizada que emerge desde lo cotidiano. Tal proceso o despertar del alumnado emerge del planteamiento de preguntas desafiantes, facilitación de recursos y apoyo en redes académicas propiciado por el profesorado. 


\section{Agradecimientos}

Al Programa de Indagación Científica para la Educación en Ciencias (ICEC) del Ministerio de Educación de Chile y a la Escuela Darío Salas Díaz.

\section{ReFERENCIAS}

Abell S., Smith D., Volkmann M. (2006) Inquiry in Science Teacher Education. En L. Flick, N. Lederman (Eds.), Scientific inquiry and the nature of science: Implications for teaching, learning, and teacher education (pp. 173-199). Dordrecht: Springer.

Agencia de Calidad de la Educación (2019) Se Puede; Quince prácticas de gestión curricular, estrategias de aula y educación integral. Santiago: Agencia de Calidad de la Educación.

Ariza M. R., Aguirre D., Quesada A., Abril A. M., García F. J. (2016) ¿Lana o metal? Una propuesta de aprendizaje por indagación para el estudio de las propiedades térmicas de materiales comunes. Revista Electrónica de Enseñanza de las Ciencias, 15(2), 297-311.

Ariza M. R. (2017) El aprendizaje por indagación: ¿existen suficientes evidencias sobre sus beneficios en la enseñanza de las ciencias? Revista Eureka sobre Enseñanza y Divulgación de las Ciencias, 14(2), 286- 299.

Bennet J., Lubben F., Hogarth S. (2007) Bringing science to life: A synthesis of the research evidence on the effects of context-based and STS approaches to science teaching. Science education, 91(3), 347-370.

Bevins S., Price G. (2016) Reconceptualising inquiry in science education. International Journal of Science Education, $38(1), 17-29$.

Busquets T., Silva M., Larrosa P. (2016) Reflexiones sobre el aprendizaje de las ciencias naturales: Nuevas aproximaciones y desafíos. Estudios pedagógicos, 42( $\mathrm{N}^{\circ}$ Especial), 117-135.

Bybee R. W. (2006) Scientific inquiry and science teaching. En L. Flick, N. Lederman (Eds.), Scientific inquiry and nature of science (pp. 1-14). Dordrecht: Springer Publishers.

Chernicoff L., Echeverría E. (2012) ¿Por qué enseñar ciencia a través de la indagación? Un caso en la Universidad Autónoma de la Ciudad de México (UACM). Educación Química, 23(4), 433-450.

Crawford B. A. (2014) From inquiry to scientific practices in the science classroom. En N. G. Lederman, S. K. Abell (Eds.). Handbook of research on science education (Vol. II) (pp. 515-544). New York: Routledge.

Furtak E. M., Seidel T., Iverson H., Briggs D. C. (2012) Experimental and Quasi-Experimental Studies of InquiryBased Science Teaching. Review of Educational Research, 82(3), 300-329.

González-Weil C., Cortéz M., Bravo P., Ibaceta Y., Cuevas K., Quiñones P., Maturana J., Abarca A. (2012) La indagación científica como enfoque pedagógico: estudio sobre las prácticas innovadoras de docentes de ciencia en EM (Región de Valparaíso). Estudios pedagógicos, 38(2), 85-102.

Hagenauer G., Hascher T., Volet S. E. (2015) Teacher emotions in the classroom: associations with students' engagement, classroom discipline and the interpersonal teacher-student relationship. European journal of psychology of education, 30(4), 385-403.

Harlen W. (Ed.) (2010) Principles and big ideas of science education. Hatfield: Association for Science Education.

Harlen W. (2015) Teaching Science for Understanding in Elementary and Middle Schools. Portsmouth: Heinemann.

Hattie J. (2009) Visible Learning, A synthesis of over 800 meta-Analyses relating to achievement. $2 .{ }^{\text {da }}$ Edición. New York: Routledge.

Hmelo-Silver, C. E., Duncan, R. G., y Chinn, C. A. (2007). Scaffolding and achievement in problem-based and inquiry learning: A response to Kirschner, Sweller, and Clark (2006). Educational Psychologist, 42(2), 99-107.

Holbrook, J., Kolodner, J. L. (2000). Scaffolding the development of an inquiry-based (science) classroom. En B. J. Fishman, S. F. O'Connor- Divelbiss (Eds.). Proceedings of the Fourth International Conference of the Learning Sciences (pp. 221-27). Ann Arbor: University of Michigan. 
Ibarra D., Ruiz J., González D., Flores J., Díaz G. (2009) Distribución espacial del pH de los suelos agrícolas de Zapopan, Jalisco, México. Agricultura técnica en México, 35(3) 267-276.

Instituto de Investigaciones Agropecuarias (INIA) (2018) Manejo Integrado de Plagas y Enfermedades Mosquita blanca.

Junta Nacional de Auxilio Escolar y Becas (JUNAEB) (s.f.) Medición de la Vulnerabilidad Multidimensional del Estudiante.

Kirschner P. A., Sweller J., Clark R. E. (2006) Why minimal guidance during instruction does not work: An analysis of the failure of constructivist, discovery, problem-based, experiential, and inquiry-based teaching. Educational Psychologist, 41(2), 75-86.

Krajcik J., Mamlok, R., Hug B. (2001) Modern content and the enterprise of science: Science education in the twentieth century. En L. Corno (Ed.), Education across a century: The centennial volume (pp. 205-238). Chicago: University of Chicago Press.

Lazonder A. W., Harmsen R. (2016) Meta-Analysis of Inquiry-Based Learning Effects of Guidance. Review of Educational Research, 20(10), 1-38.

Lederman N. G., Lederman J. S., Antink A. (2013) Nature of science and scientific inquiry as contexts for learning of science and achievement of scientific literacy. International Journal of Education in Mathematics, Science and Technology, 1(3), 138-147.

Martin-Hansen L. (2002) Defining inquiry: Exploring the many types of inquiry in the science classroom. The science teacher, 69(2), 34-37.

Mayer R. E. (2004) Should there be a three-strikes rule against pure discovery learning? The case for guided methods of instruction. American Psychologist, 59(1), 14-19.

McConney A., Oliver M. C., Woods - McConney A., Schibeci R., Maor D. (2014) Inquiry, Engagement, and Literacy in Science: A Retrospective, Cross-National Analysis Using PISA 2006. Science Education, 98(6), 963-980.

Minner D., Levy A., Century J. (2010) Inquiry-based science instruction-What is it and does it matter? Results from a research synthesis years 1984 to 2002. Journal of Research in Science Teaching, 47(4), 474-496.

National Research Council (NRC) (2012) A framework for K-12 science education: Practices, crosscutting concepts, and core ideas. Washington, D. C.: The National Academies Press.

Next Generation Science Standards (NGSS) Lead States (2013) Next Generation Sciencia Standards: For State, By State. Washington, D. C.: The National Academies Press.

Organización de las Naciones Unidas para la Alimentación y la Agricultura (FAO) (2013) Código Internacional de Conducta para la Distribución y Utilización de Plaguicidas.

Parra W., Angulo F., Soto C. A. (2018) El Vínculo del Andamiaje en la Caracterización del PCK del Profesor de Ciencias. Estudio de Caso en Educación Superior. Tecné, Episteme y Didaxis: TED, (Extraordin).

Puntambekar S., Kolodner J. L. (2005) Toward implementing distributed scaffolding: Helping students learn science from design. Journal of Research in Science Teaching: The Official Journal of the National Association for Research in Science Teaching, 42(2), 185-217.

Roberts E. B. (2007) Managing Invention and Innovation. Research-Technology Management, 50(1), 35-54.

Servicio Agrícola y Ganadero (SAG) (2004) Vigilancia fitosanitaria Aleurodicus sp. cercano a A. cocois.

Treviño E., Donoso F., Bonhomme M. (2009) ¿Cómo las escuelas chilenas pueden mejorar el aprendizaje en Ciencias? En L. Cariola, G. Cares, E. Lagos (coords), ¿Qué nos dice PISA sobre la educación de los jóvenes en Chile?: Nuevos análisis y perspectivas sobre los resultados en PISA 2006 (pp. 71-104). Santiago: Ministerio de Educación, Unidad de Currículum y Evaluación.

Windschitl M. (2003) Inquiry Projects in Science Teacher Education: What Can Investigative Experiences Reveal About Teacher Thinking and Eventual Classroom Practice? Science Education, 87(1), 112-143.

\section{INFORMACIÓN ADICIONAL}


Revista Eureka sobre Enseñanza y Divulgación de las Ciencias, 2022, vol. 19, núm. 2, Enero-Abril, I...

Para citar este articulo: Morales M., Acosta K. y Rodríguez C. (2022) El rol docente y la indagación científica: análisis de una experiencia sobre plagas en una escuela vulnerable de Chile. Revista Eureka sobre Enseñanza y Divulgación de las Ciencias 19(2), 2201. doi:10.25267/Rev_Eureka_ensen_divulg_cienc.2022.v19.i2.2201 